\title{
A Modified Predictor-Corrector Algorithm for Locating Weighted Centers in Linear Programming Y. Zhang
}

A. El-Bakry June, 1992 TR92-19 


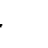




\title{
A Modified Predictor-Corrector Algorithm for Locating Weighted Centers in Linear Programming
}

\author{
Yin Zhang ${ }^{1}$ and Amr El-Bakry ${ }^{2}$ \\ March, 1992 (Revised June, 1992)
}

\begin{abstract}
In certain applications of linear programming, some particular solutions called the weighted centers of the solution set are often desired, giving rise to the need of algorithms capable of locating such centers. In this note, we modify the Mizuno-Todd-Ye predictor-corrector algorithm so that the modified algorithm is guaranteed to converge to the weighted center for given weights. The key idea is to ensure that iterates remain in a sequence of shrinking neighborhoods of the weighted central path. The modified algorithm also possesses polynomiality and superlinear convergence.
\end{abstract}

Keywords: Predictor-Corrector algorithm, weighted center of the solution set.

Abbreviated Title: A Modified Predictor-Corrector Algorithm

\footnotetext{
${ }^{1}$ Assistant Professor, Department of Mathematics and Statistics, University of Maryland Baltimore County, Baltimore, Maryland. This author's research was supported in part by NSF DMS-9102761 and DOE DE-FG05-91ER25100.

${ }^{2}$ Lecturer and Research Associate, Department of Mathematical Sciences and the Center for Research in Parallel Computation, Rice University, Houston, Texas. This author's research was supported in part by NSF Coop. Agr. No. CCR-8809615.
} 


\section{Introduction}

In some applications in economy and management sciences, it is often required to obtain particular solutions for the linear program underlying the mathematical model. One such application is the Data Envelopment Analysis (DEA) (Ref. 1) which is a method to estimate amount of inefficiencies contained in the outputs and inputs generated by managed entities called Decision Making Units. The desired solutions for the DEA linear programming model are essentially those solutions that satisfy strict complementarity. Among those strict complementary solutions, its often more beneficial to obtain a solution that is "as far as possible" from the relative boundary of the solution set (Ref. 2), i.e. it is desirable to obtain a solution that is "central" to the solution set in some sense. Several notions of centers are introduced in the literature. To cite some, we mention, the center of gravity, the center of an ellipsoid of the maximum volume inscribed inside the solution set, see (Ref. 3). Unfortunately, some of these centers are more difficult to obtain than the linear programming itself, while others can be obtained in a polynomial time but the algorithms for obtaining them are not computationally attractive (Ref. 3). Another notion of center is the analytic center of the solution set. The attractiveness of this center arise from the fact that it maximizes the product of the positive components of solutions in the relative interior of the solution set (equivalently, it maximizes the logarithmic barrier term). The analytic center can also be defined as the intersection of the central path with the solution set (Ref. 3). It is our main objective in this work to obtain an interior-point algorithm that can locate the analytic center of the solution set and also possesses both global and fast local convergence properties. Throughout this paper, the term center will be used exclusively to refer to (weighted) analytic centers, which will be defined later in this section.

We consider linear programs in the standard form:

$$
\begin{array}{ll}
\operatorname{minimize} & c^{T} x \\
\text { subject to } & A x=b, \quad x \geq 0,
\end{array}
$$


where $c, x \in \mathbf{R}^{n}, b \in \mathbf{R}^{m}, A \in \mathbf{R}^{m \times n}(m<n)$ and $A$ has full rank $m$. The dual linear program of $(1)$ is

$$
\begin{array}{ll}
\text { maximize } & b^{T} y \\
\text { subject to } & A^{T} y+z=c, \quad z \geq 0,
\end{array}
$$

where $y \in \mathbf{R}^{m}$ and $z \in \mathbf{R}^{n}$.

The optimality conditions for (1) and (2) can be written as

$$
F(x, y, z)=\left(\begin{array}{c}
A x-b \\
A^{T} y+z-c \\
X Z e
\end{array}\right)=0, \quad(x, z) \geq 0,
$$

where $X=\operatorname{diag}(x), Z=\operatorname{diag}(z)$ and $e$ is the $n$-vector of all ones.

The feasibility set of problem (3) is defined as

$$
\mathcal{F}=\left\{(x, y, z): A x=b, A^{T} y+z=c,(x, z) \geq 0\right\}
$$

A feasible point $(x, y, z) \in \mathcal{F}$ is said to be strictly feasible if $x$ and $z$ are strictly positive. In this paper we denote the set of all strictly points by $\mathcal{F}_{+}$and assume it to be nonempty.

For any point $(x, y, z) \in \mathcal{F}$, the duality gap is

$$
x^{T} z=\|F(x, y, z)\|_{1}=c^{T} x-b^{T} y \geq 0 .
$$

The equality holds only at optimal solutions. In general, the goal of a primal-dual interiorpoint method is to drive the duality gap to zero.

We denote the solution set of Problem (3) by

$$
\mathcal{S}=\{(x, y, z): F(x, y, z)=0,(x, z) \geq 0\}
$$

It is known that the existence of strictly feasible points implies that $\mathcal{S}$ is bounded. In addition, if $\mathcal{S}_{p}$ and $\mathcal{S}_{d}$ are the primal and dual solution sets, respectively, then

$$
\mathcal{S}=\mathcal{S}_{p} \times \mathcal{S}_{d}
$$


In this work, we are only concerned with the case when $\mathcal{S}$ is not a singleton set, i.e.,

$$
r i(\mathcal{S}) \neq \emptyset
$$

where $\operatorname{ri}(\mathcal{S})$ is the relative interior of $\mathcal{S}$. In this case, the solution set $\mathcal{S}$ has the following well-known structure: (i) the relative interior of $\mathcal{S}$ coincides with the set of strict complementary solutions; (ii) the zero-nonzero pattern of points in the relative interior is invariant. Therefore, for any $\left(x^{*}, y^{*}, z^{*}\right) \in \operatorname{ri}(\mathcal{S})$, the following index sets

$$
I_{x}^{+}=\left\{i: x_{i}^{*}>0,1 \leq i \leq n\right\} \text { and } I_{z}^{+}=\left\{i: z_{i}^{*}>0,1 \leq i \leq n\right\}
$$

are independent of the choice of $\left(x^{*}, y^{*}, z^{*}\right)$. Moreover, by strict complementarity

$$
I_{x}^{+} \bigcup I_{z}^{+}=\{1,2, \ldots, n\} \text { and } I_{x}^{+} \bigcap I_{z}^{+}=\emptyset
$$

Given a weight vector $w \in \mathbf{R}^{n}$ such that

$$
w>0 \text { and } e^{T} w=n
$$

the $w$-weighted center, or simply $w$-center, of the solution set $\mathcal{S}$ is defined as

$$
\left(x^{w}, y^{w}, z^{w}\right)=\arg \max \left\{\psi^{w}(x, z):(x, y, z) \in \mathcal{S}\right\}
$$

where

$$
\psi^{w}(x, z)=\prod_{i \in I_{x}^{+}} x_{i}^{w_{i}} \prod_{i \in I_{z}^{+}} z_{i}^{w_{i}}
$$

Equivalently, in (5) one can replace $\psi^{w}(x, z)$ by its logarithm, i.e.,

$$
\ln \psi^{w}(x, z)=\sum_{i \in I_{x}^{+}} w_{i} \ln x_{i}+\sum_{i \in I_{z}^{+}} w_{i} \ln z_{i}
$$

Obviously, $\left(x^{w}, y^{w}, z^{w}\right) \in \operatorname{ri}(\mathcal{S})$ and $\psi^{w}\left(x^{w}, z^{w}\right)>0$. From the structure of both $\psi^{w}(x, z)$ and $\mathcal{S}$, we see that $x^{w}$ and $\left(y^{w}, z^{w}\right)$ are the $w$-centers of the primal and the dual solution sets, respectively; namely,

$$
x^{w}=\arg \max \left\{\prod_{i \in I_{x}^{+}} x_{i}^{w_{i}}: x \in \mathcal{S}_{p}\right\} \text { and } z^{w}=\arg \max \left\{\prod_{i \in I_{z}^{+}} z_{i}^{w_{i}}:(y, z) \in \mathcal{S}_{d}\right\} .
$$


The existence and uniqueness of $w$-center can be established in a standard manner. For more details, see McLinden (Ref. 4) and Megiddo (Ref. 5).

For $w \in \mathbf{R}^{n}$ satisfying (4), the $w$-central path of Problem (3), parametrized by $\mu$, is defined as

$$
\mathcal{P}_{w}=\left\{\left(x^{w}(\mu), y^{w}(\mu), z^{w}(\mu)\right):\left(x^{w}(\mu), y^{w}(\mu), z^{w}(\mu)\right) \in \mathcal{F}_{+}, X^{w}(\mu) Z^{w}(\mu) e=\mu w, \mu>0\right\}
$$

In particular, the $w$-central path corresponding to $w=e$ is called the central path.

The existence, differentiability and limit behavior of the $w$-central paths were first studied by McLinden (Ref. 4) in a general setting, and later by Megiddo (Ref. 5) for linear programming in particular. The most striking result in McLinden (Ref. 4) is perhaps Theorem 9. In the case of linear programming, it states that the limit of the $w$-central path as $\mu$ converges to zero is the $w$-center of $\mathcal{S}$, namely,

$$
\left(x^{w}, y^{w}, z^{w}\right)=\lim _{\mu \rightarrow 0}\left(x^{w}(\mu), y^{w}(\mu), z^{w}(\mu)\right)
$$

See also Proposition 8.2 in Megiddo (Ref. 5) and the discussion preceding it.

In this paper, the norm $\|\cdot\|$ represents the Euclidean norm unless otherwise specified.

The rest of the paper is organized as follows. In Section 2 we describe the Mizuno-ToddYe predictor-corrector algorithm. We present our modified predictor-corrector algorithm in

Section 3. The convergence properties of proposed algorithm are studied in Section 4 . The final section contains concluding remarks.

\section{Predictor-Corrector Algorithm}

In this section, we describe the Mizuno-Todd-Ye predictor-corrector algorithm (Ref. 6), a primal-dual interior-point algorithm. Mizuno, Todd and Ye showed that the algorithm has an $O(\sqrt{n} L)$-iteration polynomial complexity. More recently, Ye, Güler, Tapia and Zhang (Ref. 7) showed that it also possesses $Q$-quadratic rate of convergence. 
A key ingredient in the predictor-corrector algorithm is the use of a $\beta$-neighborhood of the central path, defined for some $\beta \in(0,1 / 4]$ as

$$
\mathcal{N}(\beta)=\{(x, y, z):(x, y, z) \in \mathcal{F},\|X z-\mu e\| \leq \beta \mu\}
$$

where

$$
\mu=x^{T} z / n
$$

The Mizuno-Todd-Ye algorithm always keeps the iterates in a given $\beta$-neighborhood. Each iteration consists of two steps: a predictor step and a corrector step. The predictor step goes to a point outside the $\beta$-neighborhood but inside the $(2 \beta)$-neighborhood. Then the corrector step brings it back to a new point inside the $\beta$-neighborhood again. Both the predictor and the corrector steps satisfy the linear system

$$
\left[\begin{array}{ccc}
A & 0 & 0 \\
0 & A^{T} & I \\
Z & 0 & X
\end{array}\right]\left(\begin{array}{c}
\Delta x \\
\Delta y \\
\Delta z
\end{array}\right)=\left(\begin{array}{c}
0 \\
0 \\
r
\end{array}\right),
$$

where $r=-X z$ for the predictor step and $r=\mu e-X z$ for the corrector step. It is easy to see that the matrix in the left-hand-side of $(9)$ is $F^{\prime}(x, y, z)$, and the right-hand-side is $-F(x, y, z)$ for the predictor step and $\mu e-F(x, y, z)$ for the corrector step, noting that for any feasible point $(x, y, z)$, the first two blocks of linear equations in $F(x, y, z)=0$ are always satisfied. Hence, the predictor step is a Newton step for the system of optimality conditions $F(x, y, z)=0$, and the corrector step is a Newton step for a perturbed system.

We now formally state the Mizuno-Todd-Ye predictor-corrector algorithm.

\section{Algorithm 2.1 (Predictor-Corrector)}

Given a strictly feasible pair $\left(x^{0}, y^{0}, z^{0}\right) \in \mathcal{N}(\beta)$ for $\beta \leq 1 / 4$. For $k=0,1,2, \ldots$, do

(1) Solve for the predictor step $\left(\Delta^{p} x^{k}, \Delta^{p} y^{k}, \Delta^{p} z^{k}\right)$ from (9) with

$$
(x, z)=\left(x^{k}, z^{k}\right) \text { and } r=-X^{k} z^{k}
$$


Let $\left(\hat{x}^{k}, \hat{y}^{k}, \hat{z}^{k}\right)=\left(x^{k}, y^{k}, z^{k}\right)+\alpha^{k}\left(\Delta^{p} x^{k}, \Delta^{p} y^{k}, \Delta^{p} z^{k}\right)$, where

$$
\alpha^{k}=\max \left\{\alpha \in(0,1]:\left(x^{k}, y^{k}, z^{k}\right)+\alpha\left(\Delta^{p} x^{k}, \Delta^{p} y^{k}, \Delta^{p} z^{k}\right) \in \mathcal{N}(2 \beta)\right\}
$$

(2) Solve for the corrector step $\left(\Delta^{c} x^{k}, \Delta y^{k}, \Delta^{c} z^{k}\right)$ from (9) with

$$
(x, z)=\left(\hat{x}^{k}, \hat{z}^{k}\right), \quad r=\hat{\mu}^{k} e-\hat{X}^{k} \hat{z}^{k} \text { and } \hat{\mu}^{k}=\left(\hat{x}^{k}\right)^{T} \hat{z}^{k} / n .
$$

Let $\left(x^{k+1}, y^{k+1}, z^{k+1}\right)=\left(\hat{x}^{k}, \hat{y}^{k}, \hat{z}^{k}\right)+\left(\Delta^{c} x^{k}, \Delta y^{k}, \Delta^{c} z^{k}\right)$.

The iterates generated Algorithm 2.1 all remain in $\mathcal{N}(\beta)$ as stated in the following lemma [see (Ref. 6) for a proof].

Lemma 2.1 (Mizuno-Todd-Ye) Let $\left\{\left(x^{k}, y^{k}, z^{k}\right)\right\}$ be generated by Algorithm 2.1. Then

$$
\left\{\left(x^{k}, y^{k}, z^{k}\right)\right\} \subset \mathcal{N}(\beta) \subset \mathcal{N}(1 / 4) .
$$

From the definition of $\mathcal{N}(\beta)$, it is clear that Lemma 2.1 implies that for $\mu^{k}>0$

$$
\left\|X^{k} z^{k} / \mu^{k}-e\right\| \leq \beta \leq 1 / 4 .
$$

\section{Modified Algorithm}

When multiple solutions exist, there has been no assurance, up to the writing of this note, that the iteration sequence generated by the Mizuno-Todd-Ye predictor-corrector algorithm will converge to a solution, a center or not, as it approaches the solution set. In this section, we modify the Mizuno-Todd-Ye predictor-corrector algorithm so that the resulting algorithm is guaranteed to converge to the $w$-center of the solution set for any given weight vector $w$ satisfying (4). Throughout this paper, we will assume that a weight vector $w$ satisfies (4).

The following lemma was proved in (Ref. 8). It provides a sufficient condition for a strictly feasible sequence $\left\{\left(x^{k}, y^{k}, z^{k}\right)\right\}$ to converge to a $w$-center of the solution set. 
Lemma 3.1 (Zhang-Tapia) Let $\left\{\left(x^{k}, y^{k}, z^{k}\right)\right\} \subset \mathcal{F}_{+}$and $w$ be a weight vector. Assume

$$
\mu^{k}=\left(x^{k}\right)^{T} z^{k} / n \rightarrow 0
$$

and

$$
\left\|X^{k} z^{k} / \mu^{k}-w\right\| \rightarrow 0
$$

Then $\left\{\left(x^{k}, y^{k}, z^{k}\right)\right\}$ converges to the $w$-center of the solution set, i.e.,

$$
\lim _{k \rightarrow \infty}\left(x^{k}, y^{k}, z^{k}\right)=\left(x^{w}, y^{w}, z^{w}\right) .
$$

In order to allow the use of arbitrary weight vectors, we redefine the $\beta$-neighborhood of a $w$-central path in terms of a weight vector $w$, i.e.,

$$
\mathcal{N}_{w}(\beta)=\{(x, y, z):(x, y, z) \in \mathcal{F},\|X z-\mu w\| \leq \beta \mu \min (w)\},
$$

where $\mu=x^{T} z / n$ and $\beta \in(0,1)$.

Our approach to constructing an algorithm that is guaranteed to converge to a $w$-center is to enforce condition (11). We do so by properly choosing the steplength $\alpha^{k}$ at each iteration. The idea is to produce a sequence of gradually shrinking neighborhoods $\left\{\mathcal{N}_{w}\left(\beta^{k}\right)\right\}$ of a $w$ central path with $\beta^{k} \rightarrow 0$, and to restrict the $k$-th iterate $\left(x^{k}, y^{k}, z^{k}\right)$ to lie in $\mathcal{N}_{w}\left(\beta^{k-1}\right)$. This strategy will eventually force the iteration sequence $\left\{\left(x^{k}, y^{k}, z^{k}\right)\right\}$ to satisfy condition (11) of Lemma 3.1.

Instead of choosing $\alpha^{k}$ by (10), we use

$$
\alpha^{k}=\max \left\{\alpha \in(0,1]:\left(x^{k}, y^{k}, z^{k}\right)+\alpha\left(\Delta^{p} x^{k}, \Delta^{p} y^{k}, \Delta^{p} z^{k}\right) \in \mathcal{N}_{w}\left(2 \beta^{k}\right)\right\}
$$

where the parameter $\beta^{k} \in(0,1 / 4]$ is chosen dynamically to ensure $\beta^{k} \rightarrow 0$. It can be verified that if

$$
\left\|X^{k} z^{k}-\mu^{k} w\right\|<2 \beta^{k} \mu^{k} \min (w),
$$

then $\alpha^{k}>0$ in (13) is well defined and

$$
\left(x^{k}, y^{k}, z^{k}\right)+\alpha^{k}\left(\Delta^{p} x^{k}, \Delta^{p} y^{k}, \Delta^{p} z^{k}\right) \in \mathcal{F}_{+} .
$$


Let $\Delta^{p} X^{k}=\operatorname{diag}\left(\Delta^{p} x^{k}\right)$ and $\Delta^{c} X^{k}=\operatorname{diag}\left(\Delta^{c} x^{k}\right)$, and let

$$
\xi^{k}=\left\|\Delta^{p} X^{k} \Delta^{p^{k}}\right\| /\left(\mu^{k} \min (w)\right) .
$$

Given constants $\lambda \in(0,1)$ and $\beta \in(0,1 / 4]$, we choose $\beta^{k}$ for $k>0$ according to the following formula:

$$
\beta^{k}=\min \left(\beta, \quad 0.5\left(\xi^{k}\right)^{1-\lambda}+0.5\left\|X^{k} z^{k} / \mu^{k}-w\right\| / \min (w)\right) .
$$

Obviously, $0<\beta^{k} \leq \beta \leq 1 / 4$. Moreover, if $\left\|X^{k} z^{k}-\mu^{k} w\right\|<\beta \mu^{k} \min (w)$, then (14) is satisfied and $\alpha^{k}>0$ is well defined by (13).

We now state our modified predictor-corrector algorithm. It allows more general weight vector $w$ other than $w=e$, and it always converges to the $w$-center of the solution set $\mathcal{S}$, as will be shown in the next section.

\section{Algorithm 3.2 (Modified Predictor-Corrector)}

Let $w$ be a weight vector satisfying (4) and let $\lambda \in(0,1)$. Given a strictly feasible pair $\left(x^{0}, y^{0}, z^{0}\right) \in \mathcal{N}_{w}(\beta)$ where $\beta \leq 1 / 4$, for $k=0,1,2, \ldots$, do

(1) Solve for the predictor step $\left(\Delta^{p} x^{k}, \Delta^{p} y^{k}, \Delta^{p} z^{k}\right)$ from (9) with

$$
(x, z)=\left(x^{k}, z^{k}\right) \text { and } r=-X^{k} z^{k} .
$$

Let $\left(\hat{x}^{k}, \hat{y}^{k}, \hat{z}^{k}\right)=\left(x^{k}, y^{k}, z^{k}\right)+\alpha^{k}\left(\Delta^{p} x^{k}, \Delta^{p} y^{k}, \Delta^{p} z^{k}\right)$, where $\alpha^{k}$ is computed from (13) with $\beta^{k}$ from (16).

(2) Solve for the corrector step $\left(\Delta^{c} x^{k}, \Delta y^{k}, \Delta^{c} z^{k}\right)$ from (9) with

$$
(x, z)=\left(\hat{x}^{k}, \hat{z}^{k}\right), \quad r=\hat{\mu}^{k} w-\hat{X}^{k} \hat{z}^{k} \text { and } \hat{\mu}^{k}=\left(\hat{x}^{k}\right)^{T} \hat{z}^{k} / n .
$$

Let $\left(x^{k+1}, y^{k+1}, z^{k+1}\right)=\left(\hat{x}^{k}, \hat{y}^{k}, \hat{z}^{k}\right)+\left(\Delta^{c} x^{k}, \Delta y^{k}, \Delta^{c} z^{k}\right)$.

It should be mentioned that if $\alpha^{k}=1$ at some iteration, then $\left(\hat{x}^{k}, \hat{y}^{k}, \hat{z}^{k}\right)$ will be an optimal solution. Hence Algorithm 3.2 (and Algorithm 2.1 for that matter) can be terminated. 
However, this case seems extremely unlikely to happen in practice. Without loss of practical significance, we will assume that $\alpha^{k}<1$ for all $k$.

It is easy to verify the following well-known identities for the iteration sequence generated by Algorithm 3.2.

$$
\begin{gathered}
\mu^{k+1}=\hat{\mu}^{k}=\left(1-\alpha^{k}\right) \mu^{k} \\
\hat{X}^{k} \hat{z}^{k}-\hat{\mu}^{k} w=\left(1-\alpha^{k}\right)\left(X^{k} z^{k}-\mu^{k} w\right)+\left(\alpha^{k}\right)^{2} \Delta^{p} X^{k} \Delta^{p} z^{k} \\
X^{k+1} z^{k+1}-\mu^{k+1} w=\Delta^{c} X^{k} \Delta^{c} z^{k} .
\end{gathered}
$$

These identities will be used in analyzing the convergence properties of Algorithm 3.2 in the next section.

Analogous to Lemma 2.1 for Algorithm 2.1, we have the following lemma for Algorithm 3.2.

Lemma 3.2 Let $\left\{\left(x^{k}, y^{k}, z^{k}\right)\right\}$ be generated Algorithm 3.2. Then for $k>0$

$$
\left(x^{k}, y^{k}, z^{k}\right) \in \mathcal{N}_{w}\left(\beta^{k-1}\right) \subset \mathcal{N}_{w}(\beta) \subset \mathcal{N}_{w}(1 / 4) .
$$

Proof: By the choices of $\alpha^{k}$ and $\beta^{k}$ in (13) and (16), respectively, we have

$$
\left(\hat{x}^{k}, \hat{y}^{k}, \hat{z}^{k}\right) \in \mathcal{N}_{w}\left(2 \beta^{k}\right),
$$

which implies

$$
\left\|\hat{X}^{k} \hat{z}^{k}-\hat{\mu}^{k} w\right\| \leq 2 \beta^{k} \mu^{k} \min (w)
$$

Hence for every $i$,

$$
\left(1-2 \beta^{k}\right) \mu^{k} w_{i} \leq \hat{x}_{i}^{k} \hat{z}_{i}^{k} \leq\left(1+2 \beta^{k}\right) \mu^{k} w_{i}
$$

In particular, for all $i$

$$
\frac{1}{\hat{x}_{i}^{k} \hat{z}_{i}^{k}} \leq \frac{1}{\left(1-2 \beta^{k}\right) \mu^{k} \min (w)} .
$$

It is well-known [see (Ref. 6), for example] that for any $(\Delta x, \Delta z)$ satisfying (9)

$$
\|\Delta X \Delta z\| \leq \frac{\left\|(X Z)^{-1 / 2} r\right\|^{2}}{2} .
$$


Therefore, it follows from (19), (20) and (21) that

$$
\begin{aligned}
\left\|X^{k+1} z^{k+1}-\mu^{k+1} w\right\| & =\left\|\Delta^{c} X^{k} \Delta^{c} z^{k}\right\| \\
& \leq \|\left(\hat{X}^{k} \hat{Z}^{k}\right)^{-1 / 2}\left(\hat{X}^{k} \hat{z}^{k}-\hat{\mu}^{k} w \|^{2} / 2\right. \\
& \leq\left\|\hat{X}^{k} \hat{z}^{k}-\hat{\mu}^{k} w\right\|^{2} /\left[2\left(1-2 \beta^{k}\right) \hat{\mu}^{k} \min (w)\right] \\
& \leq\left[2 \beta^{k} \hat{\mu}^{k} \min (w)\right]^{2} /\left[2\left(1-2 \beta^{k}\right) \hat{\mu}^{k} \min (w)\right] \\
& =\left[2 \beta^{k} /\left(1-2 \beta^{k}\right)\right] \beta^{k} \hat{\mu}^{k} \min (w) \\
& \leq \beta^{k} \hat{\mu}^{k} \min (w) .
\end{aligned}
$$

In the last inequality we used the fact $\beta^{k} \leq 1 / 4$. This completes the proof.

From the definition of $\mathcal{N}_{w}(\beta)$, it is clear that Lemma 3.2 implies that for $\mu^{k}>0$

$$
\left\|X^{k} z^{k} / \mu^{k}-w\right\| / \min (w) \leq \beta^{k-1} \leq \beta \leq 1 / 4 .
$$

\section{Convergence Properties}

In this section, we demonstrate that Algorithm 3.2 possesses polynomiality and superlinear convergence. Most importantly, for any given weight vector $w$, it always converges to the $w$-center of the solution set $\mathcal{S}$. These results are given in Theorem 4.1 below, but first we need two more technical lemmas.

Lemma 4.1 Let $\left\{\alpha^{k}\right\}$ be the steplength sequence generated by Algorithm 3.2. Then

$$
\alpha^{k} \geq \frac{2}{1+\sqrt{1+4 \xi^{k} / \eta^{k}}},
$$

where $\xi^{k}$ is given by (15) and

$$
\eta^{k}=2 \beta^{k}-\left\|X^{k} z^{k} / \mu^{k}-w\right\| / \min (w) .
$$

Furthermore,

$$
\eta^{k} \geq \min \left(\beta,\left(\xi^{k}\right)^{1-\lambda}\right)
$$


Proof: If $\alpha^{k}=1,(23)$ is obviously true; so we assume $\alpha^{k}<1$. Let

$$
f^{k}(\alpha)=\left\|\left(X^{k}+\alpha \Delta^{p} X^{k}\right)\left(z^{k}+\alpha \Delta^{p} z^{k}\right)-(1-\alpha) \mu^{k} w\right\|-2(1-\alpha) \beta^{k} \mu^{k} \min (w) .
$$

It can be verified from the definition (13) of $\alpha^{k}$ that $f^{k}\left(\alpha^{k}\right)=0$. So $\alpha^{k}$ is a root of $f^{k}(\alpha)$ in $(0,1)$. Furthermore, from (16) and (22)

$$
\begin{aligned}
f^{k}(0) & =\left\|X^{k} z^{k}-\mu^{k} w\right\|-2 \beta^{k} \mu^{k} \min (w) \\
& =\max \left(\left\|X^{k} z^{k}-\mu^{k} w\right\|-2 \beta \mu^{k} \min (w),-\left(\xi^{k}\right)^{1-\lambda} \mu^{k} \min (w)\right) \\
& \leq-\min \left(\beta,\left(\xi^{k}\right)^{1-\lambda}\right) \mu^{k} \min (w) \\
& <0 .
\end{aligned}
$$

From identity (18), we have for $\alpha \in[0,1]$

$$
f^{k}(\alpha) \leq g^{k}(\alpha) \stackrel{\text { def }}{=}(1-\alpha)\left\|X^{k} z^{k}-\mu^{k} w\right\|+\alpha^{2}\left\|\Delta^{p} X^{k} \Delta^{p} z^{k}\right\|-2(1-\alpha) \beta^{k} \mu^{k} \min (w) .
$$

The quadratic function $g^{k}(\alpha)$ has a unique root, say $\gamma^{k}$, in $(0,1)$. Since $f^{k}(\alpha) \leq g^{k}(\alpha)$ in $[0,1]$ and $f^{k}(0)=g^{k}(0)<0$, we must have $\alpha^{k} \geq \gamma^{k}$. A simple calculation shows

$$
\gamma^{k}=\frac{2}{1+\sqrt{1+4 \xi^{k} / \eta^{k}}}
$$

This proves (23).

In view of (22), we have

$$
\left\|X^{k} z^{k} / \mu^{k}-w\right\| / \min (w) \leq \beta^{k-1} \leq \beta .
$$

Therefore,

$$
2 \beta-\left\|X^{k} z^{k} / \mu^{k}-w\right\| / \min (w) \geq \beta
$$

and

$$
\eta^{k}=2 \beta^{k}-\left\|X^{k} z^{k} / \mu^{k}-w\right\| / \min (w) \geq \min \left(\beta,\left(\xi^{k}\right)^{1-\lambda}\right) .
$$

This proves inequality (25) and the lemma. 
Lemma 4.2 Let the sequence $\left\{\beta^{k}\right\}$ be generated by Algorithm 3.2. Then $\left\{\beta^{k}\right\}$ converges to zero at least $Q$-linearly at the rate $1 / 2$; i.e.,

$$
\lim _{k \rightarrow \infty} \sup \frac{\beta^{k+1}}{\beta^{k}} \leq \frac{1}{2}
$$

Proof: By (22),

$$
\beta^{k} \leq \min \left(\beta, 0.5\left(\xi^{k}\right)^{1-\lambda}+0.5 \beta^{k-1}\right) .
$$

It is known [see (Ref. 7), for example] that

$$
\left\|\Delta^{p} x^{k}\right\|=O\left(\mu^{k}\right) \text { and }\left\|\Delta^{p} z^{k}\right\|=O\left(\mu^{k}\right) .
$$

This gives

$$
\xi^{k}=\left\|\Delta^{p} X^{k} \Delta^{p} z^{k}\right\| /\left(\mu^{k} \min (w)\right)=O\left(\mu^{k}\right) \rightarrow 0 .
$$

Since $\xi^{k} \rightarrow 0$ and $\beta^{k-1} \leq \beta$, we have for $k$ sufficiently large

$$
\frac{\beta^{k}}{\beta^{k-1}} \leq \frac{1}{2}+o(1)
$$

This proves the lemma.

. Now we are ready to state our main results.

Theorem 4.1 Let $\left\{\left(x^{k}, y^{k}, z^{k}\right)\right\}$ be generated Algorithm 3.2 and $\left(x^{w}, y^{w}, z^{w}\right)$ be the $w$-center of the solution set $\mathcal{S}$. Assume $\left(x^{0}\right)^{T} z^{0} \leq 2^{O(L)}$ and $\min (w)$ is independent of $n$. Then the following hold

(1) $\left(x^{k}\right)^{T} z^{k} \leq 2^{-L}$ in $O(\sqrt{n} L)$ iterations;

(2) $\left(x^{k}\right)^{T} z^{k} \rightarrow 0 Q$-superlinearly at the rate $(1+\lambda)$;

(3) $\left(x^{k}, y^{k}, z^{k}\right) \rightarrow\left(x^{w}, y^{w}, z^{w}\right) R$-superlinearly at the rate $(1+\lambda)$. 
Proof: (1) It suffices to show $\alpha^{k}=\Omega(1 / \sqrt{n})$. It is well-known that $\left\|\Delta^{p} X^{k} \Delta^{p} z^{k}\right\| \leq n \mu^{k}$, hence $\xi^{k} \leq n / \min (w)$. Furthermore, (25) implies

$$
\xi^{k} / \eta^{k} \leq \max \left(n /(\beta \min (w)),(n / \min (w))^{\lambda}\right)=O(n) .
$$

It follows from (23) that $\alpha^{k}=\Omega(1 / \sqrt{n})$.

(2) It is equivalent to prove

$$
\frac{\left(x^{k+1}\right)^{T} z^{k+1}}{\left(x^{k}\right)^{T} z^{k}}=\frac{\mu^{k+1}}{\mu^{k}}=1-\alpha^{k}=O\left(\left(\mu^{k}\right)^{\lambda}\right) .
$$

It follows from (25) and (26) that

$$
\xi^{k} / \eta^{k} \leq \max \left(\xi^{k} / \beta,\left(\xi^{k}\right)^{\lambda}\right)=O\left(\left(\mu^{k}\right)^{\lambda}\right) \rightarrow 0 .
$$

It can be readily verified from (23) [see Sec. 3 of (Ref. 7)] that

$$
1-\alpha^{k} \leq \xi^{k} / \eta^{k}=O\left(\left(\mu^{k}\right)^{\lambda}\right) .
$$

This proves (2).

(3) The convergence to the $w$-center follows directly from Lemmas $3.1,3.2$ and 4.2 . The $R$-superlinear convergence at the rate $(1+\lambda)$ follows from (2) and Hoffman's lemma (Ref. 9). This completes the proof.

\section{Concluding Remarks}

In this note, we proposed a variant of the Mizuno-Todd-Ye predictor-corrector algorithm that is guaranteed to converge to the $w$-center of the solution set $\mathcal{S}$ for any given weight vector $w$, provided that an initial point close to the $w$-central path is available. To our best knowledge, this is the first demonstration of an algorithm with such a property. The introduction of weights can be useful in applications where large values for certain variables are desirable. 
The proposed algorithm retains the $O(\sqrt{n} L)$-iteration complexity bound of the MizunoTodd-Ye predictor-corrector algorithm. On the other hand, while maintaining superlinear convergence, the proposed algorithm has a $Q$-convergence rate $(1+\lambda) \in(1,2)$ instead of quadratic.

\section{References}

1. CHARNES, A., COOPER, W.W., and THRALL, R.M. A structure for Classifying and Characterizing Efficiency and Inefficiency in Data Envelopment Analysis, The Journal of Productivity Analysis, Vol. 2, pp.197-237, 1991.

2. THRALL, R. M. Private communications, 1991.

3. GONZAGA, C. C. Path-Following Methods for Linear Programming, SIAM Reviews, Vol. 34, pp.167-224, 1992.

4. MCLINDEN, L. An analogue of Moreau's proximation theorem, with application to the nonlinear complementarity problem, Pacific J. of Math., Vol. 88, pp.101-161, 1980.

5. MEGIDDO, N. Pathways to the optimal set in linear programming, Edited by N. Megiddo, Progress in Mathematical programming, interior-point and related methods, pp.131-158. Springer-Verlag, New York, 1989.

6. MIZUNO, S., TODD, M. J. and YE, Y. On adaptive-step primal-dual interior-point algorithms for linear programming, Technical Report No. 944, School of ORIE, Cornell University, 1990.

7. YE, Y., GÜLER, O., TAPIA, R. A. and ZHANG, Y. A quadratically convergent $O(\sqrt{n} L)$-iteration algorithm for linear programming, Technical Report TR91-26, Dept. Mathematical Sciences, Rice University, 1991. 
8. ZHANG, Y. and TAPIA, R. A. On the convergence of interior-point methods to the center of solution set in linear programming, Technical Report TR91-30, Dept. Mathematical Sciences, Rice University, 1991.

9. HOFFMAN, A. J. On approximate solutions of systems of linear inequalities, Journal of Research of the National Bureau of Standards, Vol. 49, pp.263-265, 1952. 